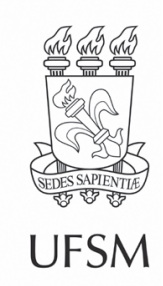

\title{
Artigos
}

\section{Potencial de seleção em uma população de Eucalyptus grandis Hill ex Maiden, para resistência à mancha foliar e ao cancro}

\author{
Selection potential in a population of Eucalyptus grandis Hill ex Maiden, \\ for resistance to leaf spot and canker
}

\author{
Eduardo Henrique Rezende ${ }^{\triangleleft}{ }^{\circ}$, Celso Garcia Auer" ${ }^{\oplus}$, Izabela Moura Duin ${ }^{\prime \prime \prime} \odot$, \\ Thiare Aparecida do Valle Coelho ${ }^{\prime \vee} \oplus$, Izabele Domingues Soares Miranda ${ }^{\bullet}$, \\ Antonio Rioyei Higa'v $\oplus$, Luciana Duque Silvav ${ }^{ }$, Álvaro Figueredo dos Santos" $" \odot$ \\ 'Universidade Federal do Tocantins, Gurupi, TO, Brasil \\ "Embrapa Florestas, Colombo, PR, Brasil \\ "'Universidade Estadual de Londrina, Londrina, PR, Brasil \\ IVUniversidade Federal do Paraná, Curitiba, PR, Brasil \\ vUniversidade Federal do Sul e Sudeste do Pará, São Félix do Xingu, PA, Brasil \\ V'Universidade de São Paulo, Escola Superior de Agricultura "Luiz de Queiroz", Piracicaba, SP, Brasil
}

\section{RESUMO}

O uso de genótipos resistentes é um dos principais métodos de controle de doenças no Brasil, sendo fundamental o melhoramento genético visando a obter novos genótipos resistentes, para suprir essa demanda. Assim, o objetivo deste trabalho foi estimar os parâmetros genéticos das variáveis resistência ao cancro, causado por Chrysoporthe cubensis e resistência à mancha foliar, causada por Cylindrocladium spp. e Kirramyces epicoccoides, bem como avaliar suas implicações na estratégia de melhoramento genético de Eucalyptus grandis. Foi instalado um teste de progênies de polinização aberta, em dois locais no Estado de São Paulo, nas regiões de Anhembi e Itatinga. O teste foi realizado em blocos casualizados, com 176 progênies e três testemunhas, uma por parcela, com 30 repetições em Itatinga e 28 repetições na região de Anhembi. A resistência ao cancro foi avaliada aos 12, 24 e 29 meses de idade na região de Anhembi e aos 15, 27 e 32 meses de idade, na região de Itatinga. A resistência à mancha foliar foi avaliada aos 12 e 24 meses de idade na região de Anhembi e aos 15 e 27 meses de idade, na região de Itatinga. Os parâmetros genéticos foram estimados, utilizando o software SELEGEN REML/BLUP®. Com a mesma população de seleção, verificou-se que, para a região de Itatinga, as estimativas de parâmetros genéticos indicam maior potencial para a seleção de genótipos resistentes ao cancro e à mancha foliar. Porém, para as condições edafoclimáticas da região de Anhembi, essa população apresenta potencial de seleção inferior, o que fica evidenciado pelos baixos valores de herdabilidade média individual de progênies estimados. Na região de Itatinga, foram obtidos valores de herdabilidade média individual de progênies para a resistência ao cancro e à mancha foliar, que variaram de moderado a alto, indicando que pode haver bons ganhos com a seleção neste local.

Palavras-Chave: Eucalyptus grandis; Seleção; Herdabilidade; Doença 


\section{ABSTRACT}

The use of resistant genotypes is one of the main methods of disease control in Brazil, the genetic improvement, aiming to obtain new genotypes that are resistant is fundamental to supply this demand. Therefore, the objective of this work was to estimate the genetic parameters of the variables: resistance to canker caused by Chrysoporthe cubensis and leaf spot caused by Cylindrocladium spp. and Kirramyces epicoccoides, and to evaluate their implications for the genetic improvement strategy of Eucalyptus grandis. An open pollination progeny test was installed in two locations in the state of São Paulo, in the regions of Anhembi and Itatinga. The test was carried out in randomized blocks, with 176 progenies and three witnesses, one per plot, with 30 repetitions in Itatinga and 28 repetitions in the Anhembi region. The canker resistance was assessed at 12, 24 and 29 months of age in the Anhembi region and at 15, 27 and 32 months of age in the Itatinga region. The resistance to leaf spot was evaluated. at 12 and 24 months of age in the Anhembi region and at 15 and 27 months of age in the Itatinga region. The genetic parameters were estimated using the SELEGEN REML/BLUP® software. It was found out, with the same selection population for the Itatinga region, estimates of genetic parameters indicate greater potential for the selection of resistant genotypes for canker and leaf spot. However, for the edaphoclimatic conditions of the Anhembi region, this population has a lower selection potential, which is evidenced by the low values of the estimated average progeny and individual heritability. In the region of Itatinga, values of average progeny and individual heritability were obtained for resistance to canker and leaf spot, which varied from moderate to high, indicating that there may be good gains with the selection in this location.

Keywords: Eucalyptus grandis; Selection; Heritability; Disease

\section{INTRODUÇÃO}

A espécie Eucalyptus grandis Hill ex Maiden ocorre naturalmente na Austrália, nas latitudes entre $16^{\circ} \mathrm{S}$ e $30^{\circ} \mathrm{S}$. A altitude natural de ocorrência varia desde o nível do mar até 1100 m. As condições climáticas dessas regiões são: precipitação de 1000 a $1750 \mathrm{~mm}$ e temperaturas entre 5 e $32{ }^{\circ} \mathrm{C}$, com clima predominantemente quente e úmido, de regiões tropicais e subtropicais (PRYOR, 1971). O crescimento desta espécie sofre influência das características físicas do solo, dos teores de argila e da matéria orgânica (PREVEDELLO et al., 2013).

Na produção de madeira, E. grandis apresenta vantagens em relação a outras espécies do mesmo gênero, pois adaptou-se melhor às condições de serraria em relação ao Eucalyptus pilularis Smith, Eucalyptus resinifera Smith e Eucalyptus cloeziana F. Muell. As características que Ihe dão essas vantagens são: apresentação de menor 
encurvamento, menor área ocupada por nós vivos e mortos, sendo que as árvores de maiores diâmetros demonstraram menores defeitos nas toras (HORNBURG et al., 2012).

O cancro e a mancha foliar são, entre outras, doenças que podem ocasionar danos ao Eucalyptus grandis, destinado à serraria no Brasil. O cancro é uma lesão margeada por calo, que afeta parte da circunferência do tronco, podendo gerar lesões profundas e reduções no diâmetro da planta e, como consequência mais grave, a mortalidade. As lesões ocasionadas pelo fungo no câmbio e no lenho danificam a madeira das árvores que perdem seu valor para o uso em serrarias, além de a produtividade também ser diretamente afetada (ALFENAS et al., 2009). Souza et al. (2010) concluíram que o volume de árvores de Eucalyptus grandis decresce significativamente com a elevação do nível de severidade de cancro.

As manchas foliares geram lesões nas folhas das árvores, ocasionando sua queda, que pode evoluir para a desfolha intensa, que reduz a capacidade da planta de realizar fotossíntese, transformando-se num desequilíbrio fisiológico e, consequentemente, na diminuição de produtividade. Em espécies de Eucalyptus com surtos de manchas foliares após a desfolha, ocorre elevada redução da biomassa em comparação a plantas sem desfolha (BARRY; PINKARD, 2013).

O cancro do eucalipto causado por Chrysoporthe cubensis é uma das mais importantes doenças no ciclo da cultura. A doença é encontrada em quase todo território nacional, com maior importância na costa do Espírito Santo, região do Vale do Rio Doce, e em certas áreas do estado de São Paulo e Minas Gerais (AUER; SANTOS; FURTADO, 2016).

A mancha foliar, por sua vez, é uma doença foliar presente em plantios de eucalipto no Brasil, desde 1973. Vários são os fungos registrados como causadores de mancha foliar no Brasil; dentre eles, temos as espécies de Cylindrocladium spp. e Kirramyces epicoccoides (ALFENAS et al., 2009). Esses fungos causam manchas foliares em várias espécies de Eucalyptus, sendo as mais suscetíveis o Eucalyptus 
urophylla, Corymbia citriodora, Eucalyptus cloeziana e Eucalyptus grandis (SANTOS; AUER; GRIGOLETTI JUNIOR, 2001).

O uso de plantas resistentes é uma medida de controle na contenção do cancro e da mancha foliar em plantios de eucalipto no Brasil. A seleção de material genético superior resistente pode ser intra e/ou interespecífica, com ou sem o uso da hibridação de espécies, para que haja a substituição de espécies utilizadas comercialmente por outras resistentes (ALFENAS et al., 2009; PASSADOR et al., 2012).

Existe grande variabilidade genética em relação a várias características dentro da espécie Eucalyptus grandis, como crescimento e tolerância à geada e a doenças. Nos estudos de Eldridge et al. (1993), os autores relataram que as plantas procedentes de Coffs Harbour apresentaram maiores taxas de crescimento em comparação com as procedentes de maiores altitudes da região sul do estado de Queensland. Porém, as procedentes de Queensland foram melhores em relação à tolerância à geada. Já as oriundas de Atherton, norte do estado de Queensland, foram mais suscetíveis a doenças fúngicas, como cancro e ferrugem, do que as de outras procedências.

No Brasil, as estratégias de melhoramento genético de Eucalyptus grandis geralmente buscam a seleção para algumas características como: volume de madeira, forma do fuste, qualidade da madeira, resistência a pragas, doenças e fatores abióticos como geadas. Essa espécie é constantemente utilizada em programas de melhoramento, além de outros que visam à hibridação com outras espécies, como é o caso do Eucalyptus urograndis, resultado do cruzamento Eucalyptus grandis x Eucalyptus urophylla (ELDRIDGE et al., 1993; FONSECA et al., 2010; REZENDE et al., 2019).

Para verificar o potencial de melhoramento numa população de plantas, um dos principais métodos é utilizar testes de progênies, que permitem a instalação de um experimento planejado, visando à avaliação da variação genética natural, em nível de espécies, procedências e progênies, permitindo assim a estimativa de parâmetros genéticos (CRUZ, 2005; RESENDE, 2015). 
Estimativas de parâmetros genéticos são extremamente importantes nos programas de melhoramento. Os principais parâmetros genéticos são a acurácia, a herdabilidade, a variância genética aditiva e a não aditiva. Destes, destaca-se a herdabilidade, que é considerada a proporção da variância total atribuída ao efeito médio dos genes. Seu valor é um bom indicativo do potencial do progresso genético de um programa de melhoramento, pois é uma das informações básicas para estimar os ganhos genéticos provenientes da seleção (RESENDE, 2015).

Diante disso, o objetivo deste trabalho foi estimar os parâmetros genéticos das variáveis resistência ao cancro causado por Chrysoporthe cubensis e resistência à mancha foliar causada por Cylindrocladium spp. e Kirramyces epicoccoides, bem como avaliar suas implicações na estratégia de melhoramento genético de Eucalyptus grandis.

\section{MATERIAL E MÉTODOS}

Foi implantado um teste de progênies nas Estações Experimentais de Ciências Florestais da Escola Superior de Agricultura "Luiz de Queiroz", da Universidade de São Paulo, na região de Anhembi $\left(22^{\circ} 47^{\prime} 22^{\prime \prime}\right.$ S, 48 $\left.07^{\prime} 38^{\prime \prime} \mathrm{W}\right)$ e na região de Itatinga $\left(23^{\circ} 06^{\prime}\right.$ 06“S, 48³6'56"W), localizados no estado de São Paulo.

O clima das duas regiões é classificado como Cfa, segundo Koppen, clima temperado úmido. Na região de Itatinga, a temperatura média é de $19,5^{\circ} \mathrm{C}$ e a precipitação média anual de 1.433,4 mm, numa altitude de 845 m, enquanto na região de Anhembi, a temperatura média é de $22,4^{\circ} \mathrm{C}$ e a precipitação média anual é de $1.336,8 \mathrm{~mm}$, numa altitude de $472 \mathrm{~m}$.

Dados meteorológicos de temperaturas média, mínima, máxima e de umidade relativa e precipitação foram coletados durante o período de realização do teste, nas estações meteorológicas localizadas nas estações experimentais, onde foram instalados os testes de progênies (Figura 1). 
Figura 1 - Climatograma da temperatura média, mínima, máxima e precipitação, no período de avaliação do teste de progênies: $(A)$ estação meteorológica na região de Anhembi-SP; (B): estação meteorológica na região de Itatinga-SP

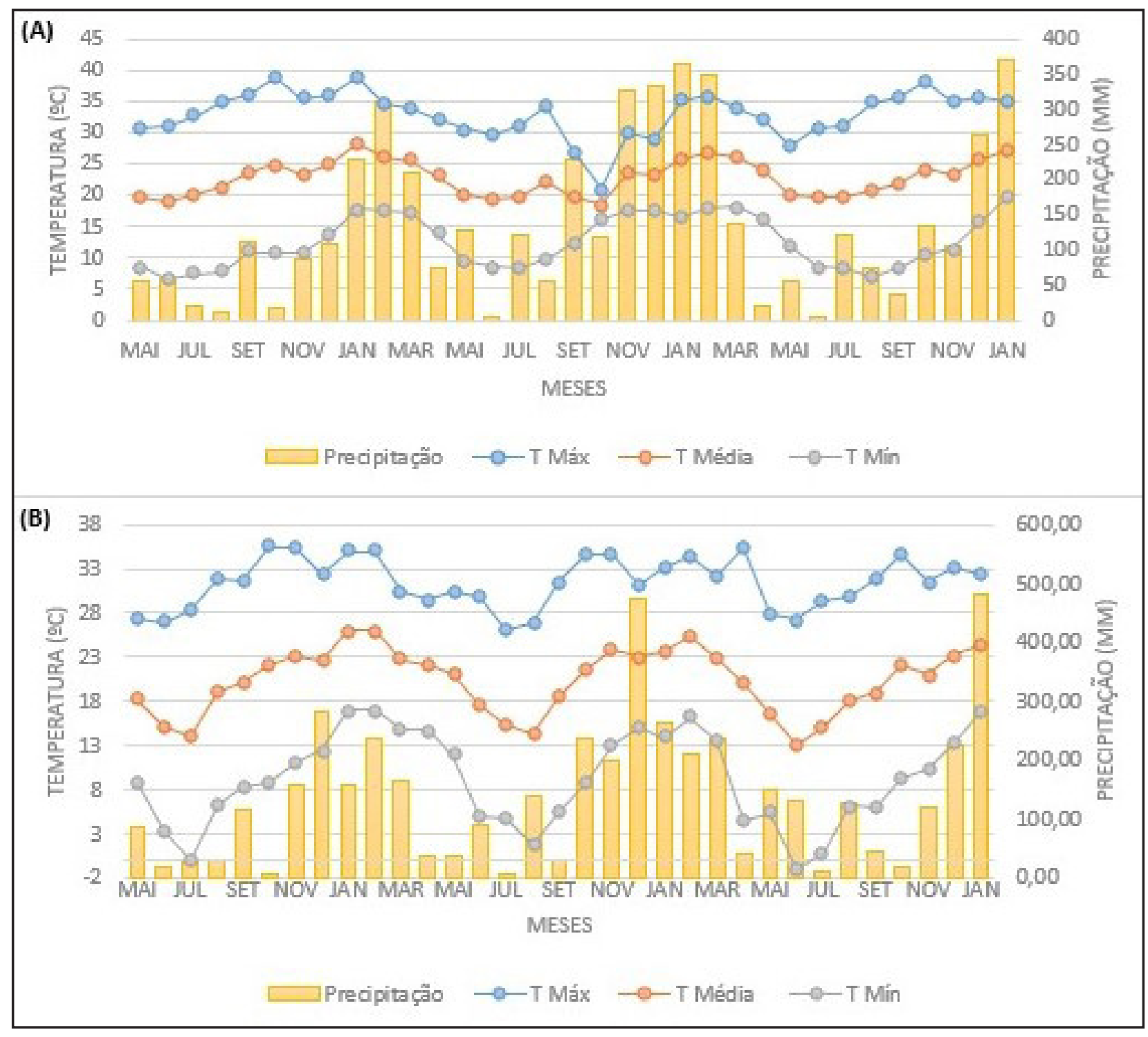

Fonte: Autores (2020)

O teste de progênies foi constituído por 176 progênies de polinização aberta de Eucalyptus grandis, oriundas de um Pomar Clonal de Sementes (PCS) no município de Itapeva, material oriundo de Atherton, Austrália. Neste teste, três testemunhas foram incluídas: duas testemunhas oriundas de sementes, sendo a primeira de um lote de sementes comerciais de Eucalyptus grandis de uma Área de Produção de Sementes 
(APS) da região de Itatinga; a outra, de um Pomar de Sementes por Mudas (PSM) de primeira geração da região de Anhembi, ambos originários de Coff's Harbour, Austrália. A terceira testemunha foi constituída por um clone comercial de Eucalyptus urophylla S. T. Blake, conhecido como AEC 144.

Foram realizadas três avaliações de cancros e duas de manchas foliares. A primeira, quando as plantas de Eucalyptus grandis estavam com 12 meses de idade em Anhembi e com 15 meses na região de Itatinga; a segunda, com 24 meses de idade na região de Anhembi e 27 meses, na de Itatinga; e a terceira avaliação, com 29 meses de idade em Anhembi e 32 meses em Itatinga.

A quantificação da severidade da mancha foliar baseou-se na escala de avaliação visual adaptada de Schultz et al. (2015) para Eucalyptus benthamii Maiden, sendo atribuídas notas de 5 a 1, de acordo com a intensidade de desfolha, sendo 5 o valor com menor severidade e 1 o valor com maior severidade da doença (Figura 2).

Figura 2 - Escala de avaliação da severidade de manchas foliares em Eucalyptus grandis

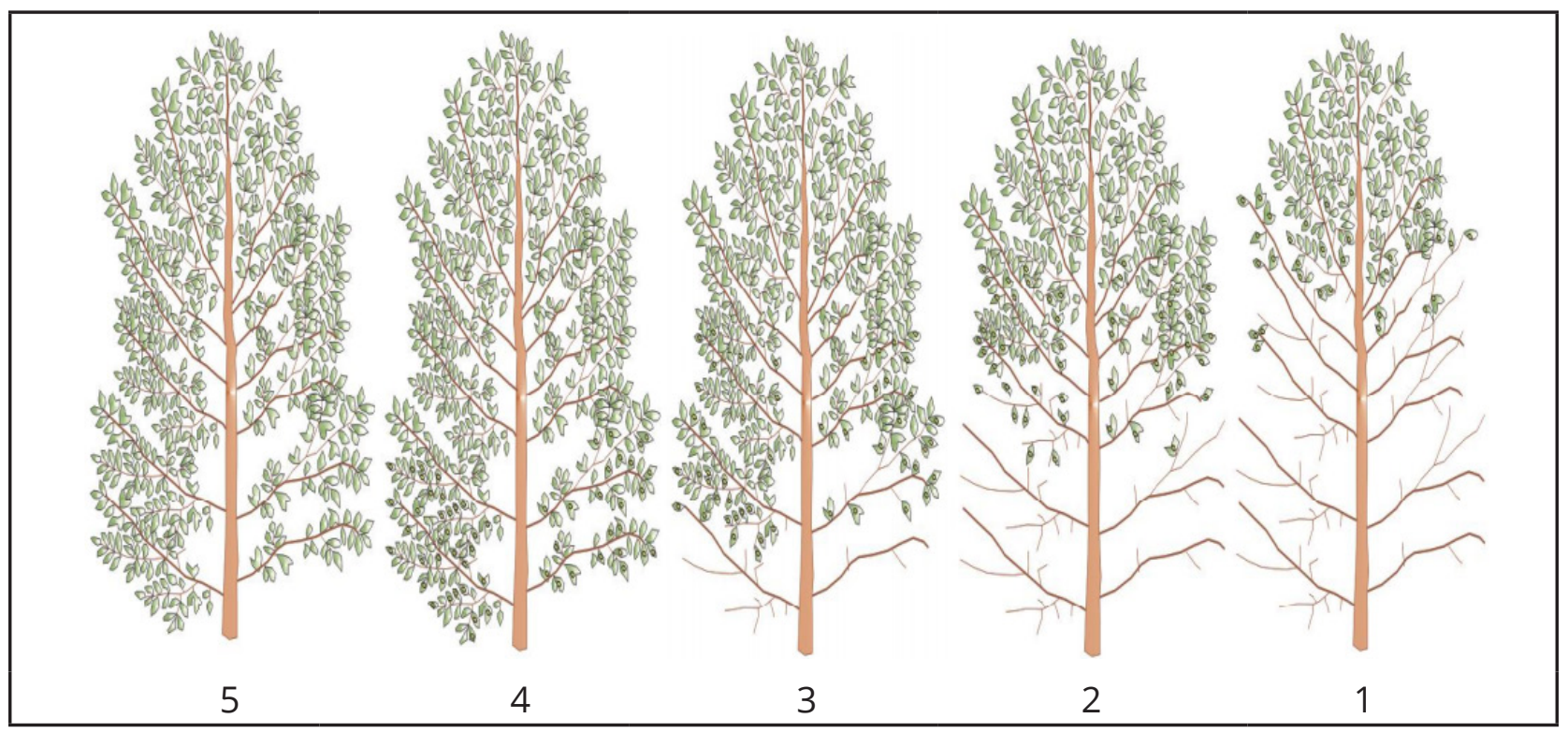

Fonte: Adaptada de Schultz et al. (2015)

Em que: Escala de notas de avaliação da severidade de manchas foliares: 5 (plantas sadias); 4 (ausência de desfolha e presença de manchas foliares nos galhos inferiores); 3 (desfolha até 1/3 da árvore); 2 (desfolha até $1 / 2$ da árvore), 1 (desfolha até 2/3 da árvore). 
Para a quantificação da severidade do cancro, foi utilizada uma escala de avaliação visual de sintomas, adaptada dos trabalhos de Oliveira e Furtado (2000) e de Souza (2008). Para tal, foram atribuídas notas de 5 a 1, de acordo com os sintomas observados nas plantas de Eucalyptus grandis (Figura 3).

Figura 3 - Escala de avaliação de severidade do cancro em Eucalyptus grandis

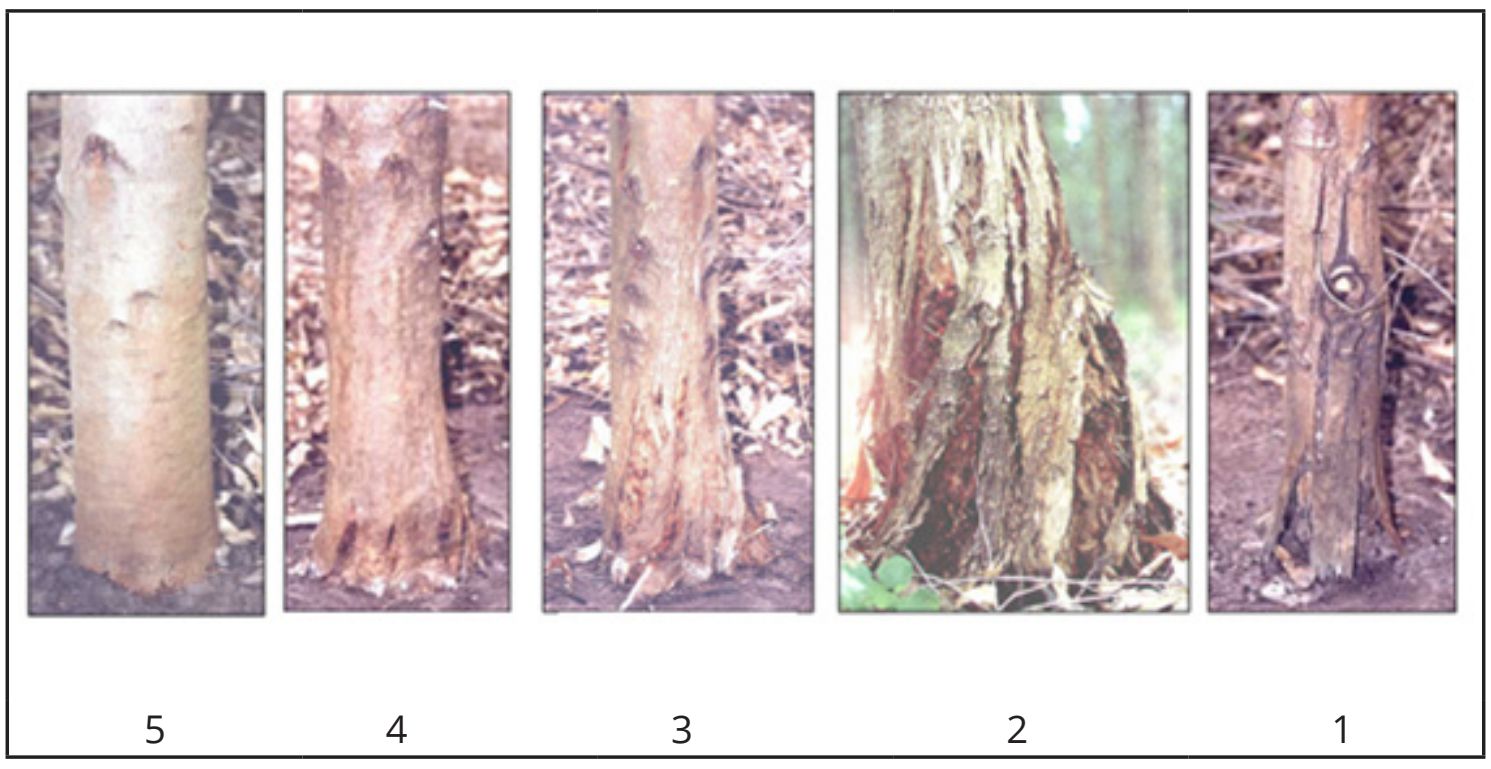

Fonte: Adaptada de Oliveira e Furtado (2000) e Souza (2008)

Em que: Escala de Notas de avaliação de severidade do cancro: 5 (Árvores sadias); 4 (Árvores com cancros superficiais, sem o comprometimento significativo da região cambial); 3 (Árvores com danos mais pronunciados, mostrando algum comprometimento da região cambial); 2 (Árvores com danos severos, comprometimento acentuado da região cambial e do lenho); 1 (Árvore morta pelo cancro).

Para a estimativa dos parâmetros genéticos, foi utilizado o software SELEGEN REML/BLUP® (RESENDE, 2002).

O modelo utilizado para a estimativa em cada local foi $y=X r+Z a+e$, em que y é o vetor de dados; r é o vetor dos efeitos de repetição (assumidos como fixos) somados à média geral; a é o vetor dos efeitos genéticos aditivos individuais (assumidos como aleatórios); e é o vetor de erros ou resíduos (aleatórios). As letras maiúsculas representam as matrizes de incidência para os referidos efeitos. Para a estimativa dos parâmetros genéticos da interação genótipo-ambiente, o modelo utilizado foi y $=\mathrm{Xr}+\mathrm{Za}+\mathrm{Wi}+\mathrm{e}$, em que y é o vetor de dados; $r$ é o vetor dos efeitos de repetição 
(assumidos como fixos) somados à média geral; a é o vetor dos efeitos genéticos aditivos individuais (assumidos como aleatórios); i é o vetor dos efeitos da interação genótipo x ambiente (aleatórios); e é o vetor de erros ou resíduos (aleatórios). As letras maiúsculas representam as matrizes de incidência para os referidos efeitos. Neste caso, o vetor $r$ contempla todas as repetições de todos os locais (ajusta combinações, repetição-local). Assim, esse vetor contempla os efeitos de locais e de repetições dentro de locais (RESENDE, 2006).

As estimativas obtidas de herdabilidade foram classificadas de acordo com Resende (2015) (Tabela 1). Os valores de acurácia considerados altos foram os maiores que 0,70 (RESENDE, 2007).

Tabela 1 - Classificação dos valores de herdabilidade

\begin{tabular}{lc}
\hline \multicolumn{1}{c}{ Valor da herdabilidade } & Classificação \\
\hline$h^{2}>0,50$ & Alta \\
$0,15<h^{2}<0,50$ & Moderada \\
$h^{2}<0,15$ & Baixa \\
\hline
\end{tabular}

Fonte: Resende (2015)

\section{RESULTADOS E DISCUSSÃO}

Na região de Anhembi, foram determinados baixos valores de herdabilidade média individual e de progênies, para todas as variáveis relacionadas ao cancro e às manchas foliares, em praticamente todas as idades estudadas (Tabela 2), exceto para a mancha foliar aos 12 meses, que apresentou 0,43 de valor de herdabilidade média de progênies e 0,10 de herdabilidade individual, revelando um baixo potencial para a seleção de indivíduos e de famílias para ambas as doenças nesta região.

O sucesso de um programa de melhoramento depende muito do potencial da população base em transmitir as características favoráveis aos seus descendentes, fato verificado pelo valor da herdabilidade, pois quanto maior o valor estimado, maiores as 
chances de ganhos com a seleção genética. Segundo Majidi, Mirlohi e Amini (2009), os valores estimados de herdabilidade indicam a resposta esperada durante o processo de seleção de populações que segregam. Os autores também ressaltam que quanto mais alto o valor da herdabilidade, maiores serão os ganhos obtidos com a seleção.

Tabela 2 - Parâmetros genéticos determinados para as variáveis resistência ao cancro e à mancha foliar das progênies de Eucalyptus grandis, em diferentes idades, na região Anhembi-SP

\begin{tabular}{|c|c|c|c|c|c|}
\hline \multirow{2}{*}{$\begin{array}{l}\text { Parâmetros } \\
\text { genéticos }\end{array}$} & \multicolumn{3}{|c|}{ Cancro/Idade (mês) } & \multicolumn{2}{|c|}{ Mancha foliar/ldade (mês) } \\
\hline & 12 meses & 24 meses & 29 meses & 12 meses & 24 meses \\
\hline$\sigma_{a^{2}}$ & 0,002 & 0,005 & 0,009 & 0,086 & 0,001 \\
\hline$\sigma_{\mathrm{e}^{2}}$ & 0,404 & 1,909 & 2,520 & 0,717 & 0,444 \\
\hline$\sigma_{f^{2}}$ & 0,406 & 1,914 & 2,530 & 0,804 & 0,446 \\
\hline$h^{2}{ }_{a}$ & $0,005 \pm 0,006$ & $0,002 \pm 0,004$ & $0,003 \pm 0,005$ & $0,108 \pm 0,026$ & $0,003 \pm 0,005$ \\
\hline$h_{m p}^{2}$ & 0,037 & 0,019 & 0,026 & 0,437 & 0,022 \\
\hline$h^{2}{ }_{a d}$ & 0,004 & 0,002 & 0,002 & 0,083 & 0,002 \\
\hline$r_{\text {аа }}$ & 0,193 & 0,141 & 0,163 & 0,661 & 0,151 \\
\hline $\mathrm{CV}_{\mathrm{gi}} \%$ & 0,972 & 1,72 & 2,637 & 9,492 & 1,914 \\
\hline $\mathrm{CV}_{\mathrm{gp}} \%$ & 0,486 & 0,861 & 1,318 & 4,746 & 0,957 \\
\hline $\mathrm{CV}_{\mathrm{e}} \%$ & 13,052 & 31,968 & 42,222 & 28,487 & 33,116 \\
\hline Média geral & 4,880 & 4,326 & 3,765 & 3,105 & 2,016 \\
\hline
\end{tabular}

Fonte: Autores (2020)

Em que: $\sigma_{a^{2}}=$ variância genética aditiva; $\sigma_{e^{2}}=$ variância residual; $\sigma_{f^{2}}=$ variância fenotípica individual; $\mathrm{h}_{\mathrm{a}}{ }_{\mathrm{a}}=$ herdabilidade individual no sentido restrito; $\mathrm{h}_{\mathrm{mp}}^{2}=$ herdabilidade da média de progênie; $\mathrm{r}_{\mathrm{aa}}=$ acurácia da seleção de progênie; $\mathrm{h}^{2}{ }_{\mathrm{ad}}=$ herdabilidade aditiva dentro de progênie; $\mathrm{CV}_{\mathrm{gi}} \%$ = coeficiente de variação genética aditiva individual; $\mathrm{CV}_{\mathrm{gp}} \%$ = coeficiente de variação genética entre progênies; $\mathrm{CV}_{\mathrm{e}} \%=$ coeficiente de variação residual.

Quando o valor de herdabilidade é baixo, não é indicada a seleção baseada na característica, devido ao baixo controle genético. Silva (2008) não recomenda a seleção baseada na variável bifurcação em progênies de Eucalyptus benthamii, selecionadas para a resistência à geada, devido aos baixos valores de herdabilidade encontrados, que variam entre $0,03 \%$ e $6 \%$.

Em um estudo realizado por Miranda et al. (2013), foram verificadas diferenças 
entre os valores estimados de herdabilidade para a variável diâmetro à altura do peito - DAP, em avaliação realizada na região de Itatinga, em comparação com a região de Anhembi, para a mesma população. Maiores valores de herdabilidade também foram relatados por Silva et al. (2013), na seleção para resistência à ferrugem de Puccinia psidii em Eucalyptus grandis, no Estado de São Paulo.

Os valores de acurácia obtidos nas análises de parâmetros genéticos em progênies na região de Anhembi foram considerados baixos, de acordo com Resende (2007), exceto para a variável mancha foliar aos 12 meses, que apresentou valor alto de acurácia, com 0,66 (Tabela 2). Esse parâmetro genético está associado à precisão do processo de seleção, sendo um dos fatores mais relevantes para a potencialização dos ganhos genéticos. De maneira geral, os valores genéticos preditos não são iguais aos valores genéticos verdadeiros dos indivíduos. A proximidade entre esses dois valores pode ser avaliada com base na acurácia (VAN VLECK; POLLAK; OLTENACU, 1987), que se refere à correlação entre os valores genéticos preditos e os valores genéticos verdadeiros. Quanto maior a acurácia na avaliação de um indivíduo, maior é a confiança na avaliação e no valor genético predito desse indivíduo.

No teste de progênies na região de Itatinga, as estimativas de herdabilidade média de progênies variaram entre 0,60 e 0,68, e foram considerados altos, exceto para a variável mancha foliar aos 27 meses, que apresentou herdabilidade moderada de 0,42 . Os valores de herdabilidade individuais variaram entre 0,19 e 0,27, para todas as variáveis, sendo considerados moderados, exceto para mancha foliar aos 27 meses, com baixo valor de herdabilidade de 0,09 (Tabela 3).

Uma questão que deve ser levantada com relação aos parâmetros genéticos determinados ao longo das idades das árvores diz respeito à fenologia do eucalipto e seus efeitos na incidência e na severidade de doenças. A epidemia da mancha foliar e do cancro podem comportar-se de modo bem diferente em função da idade e da fenologia das plantas. A mancha foliar de Cylindrocladium candelabrum em Eucalyptus benthamii apresentou-se mais severa em plantios mais jovens, inclusive com desfolha, 
em função dos tecidos jovens das folhas, que, com a passagem para a fase adulta, tornam-se mais resistentes (SCHULTZ et al., 2015). Ao contrário, o cancro do eucalipto pode começar a atacar a partir dos seis meses e vai aumentando a incidência e, posteriormente, a severidade, num continuum, dependendo da suscetibilidade do material genético (ALFENAS et al., 2009). Esses aspectos temporais podem explicar parte da variação identificada nos parâmetros genéticos encontrados, quando se espera que os valores de acurácia para a variável mancha foliar sejam maiores em idades mais jovens (entre primeiro e segundo ano), enquanto, para a variável cancro, seja necessário avaliá-los em idades mais avançadas, no caso, a partir do terceiro ano. Outro aspecto do cancro é a maior ocorrência em plantios seminais (ALFENAS et al., 2009), como é o caso deste estudo com as progênies de Eucalyptus grandis.

Tabela 3 - Parâmetros genéticos determinados para as variáveis resistência ao cancro e mancha foliar, das progênies de Eucalyptus grandis, em diferentes idades, na região de Itatinga-SP

\begin{tabular}{|c|c|c|c|c|c|}
\hline \multirow{2}{*}{$\begin{array}{c}\text { Parâmetros } \\
\text { genéticos }\end{array}$} & \multicolumn{3}{|c|}{ Cancro/Idade (mês) } & \multicolumn{2}{|c|}{ Mancha foliar/ldade (mês) } \\
\hline & 15 & 27 & 32 & 15 & 27 \\
\hline$\sigma_{a^{2}}$ & 0,136 & 0,565 & 0,509 & 0,073 & 0,037 \\
\hline$\sigma_{\mathrm{e}^{2}}$ & 0,488 & 1,509 & 1,882 & 0,307 & 0,359 \\
\hline$\sigma_{f^{2}}$ & 0,624 & 2,075 & 2,392 & 0,381 & 0,396 \\
\hline$h^{2}{ }_{a}$ & $0,217 \pm 0,038$ & $0,272 \pm 0,042$ & $0,212 \pm 0,037$ & $0,194 \pm 0,036$ & $0,094 \pm 0,036$ \\
\hline $\mathrm{h}_{\mathrm{mp}}^{2}$ & 0,633 & 0,687 & 0,627 & 0,604 & 0,421 \\
\hline$h^{2}{ }_{a d}$ & 0,172 & 0,219 & 0,168 & 0,153 & 0,072 \\
\hline$r_{\text {аa }}$ & 0,795 & 0,828 & 0,792 & 0,777 & 0,649 \\
\hline $\mathrm{CV}_{\mathrm{gi}} \%$ & 7,656 & 17,482 & 18,820 & 11,728 & 11,239 \\
\hline $\mathrm{CV}_{\mathrm{gp}} \%$ & 3,828 & 8,741 & 9,410 & 5,864 & 5,619 \\
\hline $\mathrm{CV}_{\mathrm{e}} \%$ & 15,957 & 32,313 & 39,681 & 25,964 & 36,069 \\
\hline Média geral & 4,816 & 4,303 & 3,792 & 2,319 & 1,725 \\
\hline
\end{tabular}

Fonte: Autores (2020)

Em que: $\sigma_{a^{2}}=$ variância genética aditiva; $\sigma_{e^{2}}=$ variância residual; $\sigma_{f^{2}}=$ variância fenotípica individual; $\mathrm{h}^{2}{ }_{\mathrm{a}}=$ herdabilidade individual no sentido restrito; $\mathrm{h}_{\mathrm{mp}}^{2}=$ herdabilidade da média de progênie; $\mathrm{r}_{\mathrm{aa}}=$ acurácia da seleção de progênie; $\mathrm{h} 2_{\mathrm{ad}}=$ herdabilidade aditiva dentro de progênie; $\mathrm{CV}_{\mathrm{gi}} \%=$ coeficiente de variação genética aditiva individual; $\mathrm{CV}_{\mathrm{gp}} \%$ = coeficiente de variação genética entre progênies; $\mathrm{CV}_{\mathrm{e}} \%$ = coeficiente de variação residual. 
Os valores de acurácia na região de Itatinga variaram entre 0,77 e 0,83, sendo sempre superiores a 0,70, exceto para a mancha foliar aos 27 meses, que apresentou acurácia seletiva um pouco menor de 0,65 (Tabela 3).

Os valores de herdabilidade e acurácia obtidos indicam que, na região de Itatinga, é possível fazer seleção genética de famílias e de indivíduos para todas as variáveis, com possibilidades de bons ganhos genéticos com a seleção individual e entre famílias, para as variáveis estudadas. Na região de Itatinga, as estimativas de herdabilidade em nível de famílias e individual foram maiores, possivelmente, devido à influência do ambiente na população, proporcionando resultados diferentes de herdabilidade para as progênies de Eucalyptus grandis. A ocorrência do cancro é maior em regiões tropicais, com precipitação acima de 1200 mm (ALFENAS et al., 2009). Itatinga, que apresenta condições de precipitação maiores do que Anhembi, pode explicar parte dessa variação. A estimativa de herdabilidade é um parâmetro implícito a uma população e, ao mudar o material genético, o ambiente e o tempo, os valores de herdabilidade tendem a ser diferentes (RESENDE, 2015).

Poucos estudos de campo apresentam estimativas de parâmetros genéticos específicos para mancha foliar. A variabilidade genética para a resistência à mancha foliar foi observada entre espécies e progênies de Eucalyptus, em testes com observações em campo e com inoculações do patógeno (FONSECA et al., 2010). Em outra mancha foliar ocasionada por Teratosphaeria nubilosa em Eucalyptus globulus, Balmelli et al. (2013) encontraram valores de herdabilidade individual de 0,40 como índice de dano foliar.

Valores semelhantes de herdabilidade aos encontrados na região de Itatinga foram observados por outros autores em relação à mancha foliar ocasionada por outros fungos em eucalipto. Para a mancha Teratosphaeria, Potts et al. (2004) verificaram valor de 0,60 para severidade de Teratosphaeria nubilosa; Dungey et al. (1997), entre 0,12 e 0,21 para Teratosphaeria nubilosa e Teratosphaeria cryptica; Carnegie e Ades (2005), entre 0,17 e 0,36 para Teratosphaeria cryptica; Balmelli et al. (2013) encontraram valores de herdabilidade individual de 0,40 para o índice de dano foliar causado por T. nubilosa. 
Também para o cancro são poucos os estudos, entre eles, o de Borges e Brune (1981), com resistência de Eucalyptus grandis ao cancro de Diaporthe cubensis Bruner (=Chrysoporthe cubensis). Os autores verificaram valores de herdabilidade entre 0,65 e 0,77. Esses resultados são superiores aos encontrados na região de Anhembi neste trabalho, possivelmente, porque, naquela época, o material genético estudado pelos autores possuía maior variabilidade genética. Por outro lado, Martins, Martins e Pinho (2006) encontraram valor de herdabilidade média de progênies de 0,59 para a resistência ao cancro em progênies de Eucalyptus grandis, abaixo dos valores do presente estudo.

Os valores de correlação genotípica entre o desempenho nas duas regiões variaram de 0,05 a 0,51 (Tabela 4). Resende (2007) relata que valores de correlação maiores de 0,67, entre diferentes locais, são considerados adequados para a utilização de uma única zona de seleção e melhoramento. Assim, as melhores progênies na região de Anhembi não foram as melhores na região de Itatinga, ou seja, será necessário estabelecer uma zona de melhoramento em cada região.

Esses resultados são semelhantes aos encontrados por Oliveira (2017), que observou correlação de 0,61 a 0,54 para a sobrevivência e a forma do fuste de Eucalyptus grandis; porém, o mesmo autor identificou interação genótipo-ambiente alta para as variáveis grau de infecção à ferrugem, DAP e bifurcação. Miranda et al. (2013) também encontraram altos valores, maiores que 0,90 de interação, entre diferentes locais, para a resistência à ferrugem em Eucalyptus grandis.

Não havendo interação genótipo-ambiente significativa, a indicação é que o processo de melhoramento deve ser realizado em duas zonas, pois as melhores progênies num local não são as melhores no outro local (RESENDE, 2007), Porém, como para a região de Anhembi as estimativas indicam baixa herdabilidade para as variáveis relacionadas a manchas foliares e ao cancro, sugere-se que essa população base estudada seja foco de programas de melhoramento genético para a seleção de genótipos resistentes a manchas foliares e ao cancro, apenas para a região de Itatinga. 
Tabela 4 - Comparação dos parâmetros genéticos da interação genótipo x ambiente, para as variáveis cancro e mancha foliar do teste de progênies de Eucalyptus grandis nas regiões de Anhembi-SP e de Itatinga-SP

\begin{tabular}{|c|c|c|c|c|c|}
\hline $\begin{array}{l}\text { Parâmetros } \\
\text { genéticos }\end{array}$ & $\begin{array}{c}\text { Cancro } \\
1^{\circ} \text { Avaliação }\end{array}$ & $\begin{array}{c}\text { Cancro } \\
2^{\circ} \text { Avaliação }\end{array}$ & $\begin{array}{c}\text { Cancro } \\
3^{\circ} \text { Avaliação }\end{array}$ & $\begin{array}{c}\text { Mancha } \\
1^{\circ} \text { Avaliação }\end{array}$ & $\begin{array}{c}\text { Mancha } \\
2^{\circ} \text { Avaliação }\end{array}$ \\
\hline$\sigma^{2}{ }_{i n t}$ & 0,008 & 0,013 & 0,041 & 0,031 & 0,002 \\
\hline$\sigma_{e}^{2}$ & 0,001 & 0,011 & 0,044 & 0,008 & 0,009 \\
\hline$\sigma_{a}^{2}$ & 1,134 & 1,399 & 2,350 & 0,910 & 0,484 \\
\hline$\sigma_{f}^{2}$ & 1,144 & 1,424 & 2,435 & 0,950 & 0,496 \\
\hline$h^{2}{ }_{a}$ & $0,007 \pm 0,005$ & $0,009 \pm 0,005$ & $0,016 \pm 0,007$ & $0,032 \pm 0,010$ & $0,004 \pm 0,004$ \\
\hline $\mathrm{c}^{2} \mathrm{i}_{\mathrm{nt}}$ & 0,001 & 0,007 & 0,018 & 0,009 & 0,019 \\
\hline$r_{\text {gloc }}$ & 0,515 & 0,231 & 0,189 & 0,474 & 0,055 \\
\hline Média geral & 4,621 & 4,512 & 3,830 & 2,715 & 1,962 \\
\hline
\end{tabular}

Fonte: Autores (2020)

Em que: $\sigma_{\text {int }}^{2}=$ variância genética aditiva; $\sigma^{2}=$ variância da interação genótipo x ambiente; $\sigma^{2}{ }_{a}=$ variância residual; $\sigma_{f}^{2}=$ variância fenotípica individual; $h^{2}{ }_{a}=$ herdabilidade individual no sentido restrito; $c^{2}{ }_{\text {int }}=$ coeficiente de determinação dos efeitos da interação genótipo $x$ ambiente; $r_{\text {gloc }}=$ correlação genotípica entre o desempenho nos vários ambientes.

Desse modo, a seleção deverá ocorrer somente na região de Itatinga, utilizando as idades em que as variáveis apresentaram melhor controle e variabilidade genética, expressa pelos maiores valores de herdabilidade, bem como utilizando as idades de avaliação que possibilitem a seleção precoce das plantas, com ganhos no tempo de seleção. Sendo assim, recomenda-se a seleção para a variável cancro, aos 27 meses e para mancha foliar, aos 15 meses.

\section{CONCLUSÕES}

O estudo demonstra que uma mesma população de Eucalyptus. grandis tem potencial para ser utilizada como população base para o desenvolvimento de programa de melhoramento genético, que vise à seleção de genótipos mais resistentes a manchas foliares e ao cancro, na região de Itatinga, porém não apresenta o mesmo potencial na região de Anhembi. Fica evidenciado também a importância da fenofase da planta no momento da fenotipagem para o sucesso da seleção. A seleção na região de Itatinga pode ser feita na idade de 15 meses para a variável mancha foliar e para o cancro do eucalipto, aos 27 meses. 


\section{AGRADECIMENTOS}

Agradecimentos à professora Luciana Duque Silva e a toda equipe das Estações Experimentais de Engenharia Florestal, à Escola Superior de Agricultura Luiz de Queiroz, de Anhembi-SP e de Itatinga-SP, pelo apoio na condução e na manutenção dos experimentos de campo deste trabalho.

\section{REFERÊNCIAS}

ALFENAS, A. C. et al. Clonagem e doenças do eucalipto. 2. ed. Viçosa, MG: [s. n.], 2009. 500 p.

AUER, C. G.; SANTOS, A. F dos; FURTADO, E. L. Doenças do eucalipto. In: AMORIM, L. et al. Manual de fitopatologia. 5. ed. Ouro Fino: Editora Agronômica Ceres, 2016. v. 2. p. 359-372.

BALMELLI, G. et al. Mejoramento genético em Eucalyptus globulus y Eucalyptus maidenii por resistência a Teratosphaeria nubilosa. In: JORNADA TÉCNICA DE PROTECCIÓN FORESTAL, AVANCES DE INVESTIGACIÓN EM PLAGAS Y ENFERMIDADES FORESTALES, 5., 2013. Anales [...]. [S. I.: s. n.], 2013. p. 55-65.

BARRY, K. M.; PINKARD, E. A. Growth and photosynthetic responses following defoliation and bud removal in eucalypts. Forest Ecology and Management, [s. I.], n. 293, p. 9-16. abr./jun. 2013.

BORGES, R. C. G.; BRUNE, A. Estudo quanto à resistência à Diaporthe cubensis Bruner em Eucalyptus grandis W Hill ex. Maiden. Revista Árvore, Viçosa, MG, v. 5, n. 1, p. 115-120, jan./ mar. 1981.

CARNEGIE, A. J.; ADES, P. K. Variation in Eucalyptus globulus Labill. and E. nitens Dean and Maiden in susceptibility of adult foliage to disease caused by Mycosphaerella cryptica (Cooke) Hansf. Silvae Genetica, [s. I.], v. 54, n. 4, p. 174-184, dez. 2005.

CRUZ, C. D. Princípios de genética quantitativa. Viçosa, MG: UFV, 2005. 394 p.

DUNGEY, H. S. et al. Mycosphaerella leaf disease: genetic variation in damage to Eucalyptus nitens, Eucalyptus globulus, and their F-1 hybrid. Canadian Journal of Forest Research, [s. I.], v. 27, p. 750-759, 1997.

ELDRIDGE, K. et al. Eucalypt domestication and breeding. New York: Oxford University Press, 1993. $288 \mathrm{p}$.

FONSECA, S. M. et al. Manual prático de melhoramento genético do eucalipto. Viçosa, MG: Editora UFV, 2010. 200 p.

HORNBURG U. K. F. et al. Qualidade das toras e da madeira serrada de seis espécies de eucalipto cultivadas no litoral de Santa Catarina. Scientia Forestalis, Piracicaba, v. 40, n. 96, p. 463-471, dez. 2012.

MAJIDI, M. M.; MIRLOHI, A.; AMINI, F. Genetic variation heritability and correlation of agromorphological traits in tall fescue (Festuca arundinaceae Schreb.). Euphytica, [s. I.], v. 167, n. 3, p. 323-331, jan. 2009. 
MARTINS, I. S.; MARTINS, R. C. C.; PINHO, D. Alternativas de índices de seleção em uma população de Eucalyptus grandis Hill ex Maiden. Cerne, Lavras, v. 12, n. 3, p. 287-291, jul./set. 2006.

MIRANDA, A. C. et al. Heritability for resistance to Puccinia psidii Winter rust in Eucalyptus grandis Hill ex Maiden in Southwestern Brazil. Tree Genetics \& Genomes, [s. I.], v. 9, n. 2, p. 321-329, abr. 2013.

OLIVEIRA, R. R.; FURTADO, E. L. Escala diagramática com categorias de severidade de ataque para o cancro basal do Eucalipto. In: SEMINÁRIO DE INICIAÇÃO CIENTÍFICA FCA/UNESP, 2000, São Paulo. Resumos [...]. São Paulo: FCA; UNESP, 2000.

OLIVEIRA, T. W. B. Seleção simultânea para crescimento, grau de infecção à ferrugem e qualidade da madeira no melhoramento de Eucalyptus grandis Hill ex Maiden. Dissertação (Mestrado) - Escola Superior de Agricultura Luiz de Queiroz, Piracicaba, 2017.

PASSADOR, M. M. et al. Teratosphaeria nubilosa em plantações comerciais de Eucalyptus globulus nas regiões Sul e Sudeste do Brasil. Summa Phytopathologica, Botucatu, v. 38, n. 1, p .11-16, jan./mar. 2012.

POTTS, B. M. et al. Quantitative genetic control of Mycosphaerella resistance. In: EUCALYPTUS GLOBULUS AND IMPACT ON GROWTH, 2004, Aveiro. Proceedings [...]. Aveiro: [s. n.], 2004. p. 46-61.

PREVEDELLO, J. et al. Manejo do solo e crescimento inicial de Eucalyptus grandis Hill ex Maiden em Argissolo. Ciência Florestal, Santa Maria, v. 23, n. 1, p. 129-138, jan./mar. 2013.

PRYOR, L. D. A classification of the eucalypts. Canberra: Australian National University, 1971. $102 \mathrm{p}$.

RESENDE, M. D. V. Genética biométrica e estatística no melhoramento de plantas perenes. Brasília: Embrapa Informação Tecnológica, 2002. 975 p.

RESENDE, M. D. V. Genética quantitativa e de populações. Viçosa, MG: Suprema, 2015. 463 p.

RESENDE, M.D.V. Matemática e estatística na análise de experimentos e no melhoramento genético. Colombo: Embrapa Florestas, 2007. 561 p.

RESENDE, M. D. V. Software Selegen-REML/BLUP. Manual do Usuário. Campo Grande: Embrapa, 2006. 305 p.

REZENDE, E. H. et al. Avaliação da resistência de progênies de Eucalyptus grandis para mancha foliar de Cylindrocladium e Kirramyces. Summa Phytopathologica, Botucatu, v. 45, n. 3, p. 295-301, out. 2019.

SANTOS, A. F dos.; AUER, C. G.; GRIGOLETTI JUNIOR, A. Doenças do eucalipto no sul do Brasil: Identificação e controle. [S. I.], Embrapa Florestas, 2001. 20 p. (Circular Técnica, 45). 
SCHULTZ, B. et al. Impacto da mancha foliar causada por Cylindrocladium candelabrum em plantios jovens de Eucalyptus benthamii em Rio Negrinho-SC. Ciência Florestal, Santa Maria, v. 25, p. 307-316, abr./jun. 2015.

SILVA, L. D. Melhoramento genético de Eucalyptus benthamii Maiden et Cambage visando à produção de madeira serrada em áreas de ocorrência de geadas severas. Tese (Doutorado) - Universidade Federal do Paraná, Curitiba, 2008. 256 p.

SILVA, P. H. M. et al. Selecting for rust (Puccinia psidii) resistance in Eucalyptus grandis in Sao Paulo State, Brazil. Forest Ecology and Management, [s. I.], v. 303, p. 91-97, 2013.

SOUZA, H. G. Resistência do eucalipto ao cancro de Chrysoporthe cubensis e Botryosphaeria sp. Dissertação (Mestrado em Ciências Agronômicas) - Universidade Estadual Paulista, São Paulo, 2008.

SOUZA, S. E. et al. Influência do cancro basal em Eucalyptus grandis nas propriedades da madeira e polpação kraft. Scientia Forestalis, Piracicaba, v. 38, n. 88, p. 547-557, dez. 2010.

VAN VLECK, L. D.; POLLAK, E. J.; OLTENACU, E. A. B. Genetics for the animal sciences. New York: W. H. Freeman, 1987. 391 p.

\section{Contribuição de Autoria}

\section{1 - Eduardo Henrique Rezende}

Engenheiro Florestal, Dr.

https://orcid.org/0000-0002-3150-8793• eduardorezende114@gmail.com

Contribuição: Escrita - primeira redação, Escrita - revisão e edição, Conceituação,

Curadoria de dados, Análise Formal, Investigação, Metodologia

\section{2 - Celso Garcia Auer}

Engenheiro Florestal, Dr., Pesquisador

https://orcid.org/0000-0002-4916-2460 • celso.auer@gmail.com

Contribuição: Conceituação, Análise Formal, Metodologia, Supervisão, Validação,

Escrita - revisão e edição

\section{3 - Izabela Moura Duin}

Engenheira Florestal, Ma., Doutoranda

https://orcid.org/0000-0003-0035-9630• izabeladuin@gmail.com

Contribuição: Investigação, Visualização de dados 


\section{4 - Thiare Aparecida do Valle Coelho}

Engenheira Florestal, Ma.

https://orcid.org/0000-0003-0528-5079•ccelho.thiare@gmail.com

Contribuição: Investigação, Visualização de dados

\section{5 - Izabele Domingues Soarez}

Engenheira Florestal Dra., Professora

https://orcid.org/0000-0001-6867-1159•izabele.soares@gmail.com

Contribuição: Investigação, Visualização de dados

\section{6 - Antonio Rioyei Higa}

Engenheiro Florestal, Dr.

https://orcid.org/0000-0001-8486-0611 •antonio.higa@gmail.com

Contribuição: Conceituação, Análise Formal, Metodologia, Supervisão, Validação, Escrita - revisão e edição

\section{7 - Luciana Duque Silva}

Engenheira Florestal, Dra., Professora

https://orcid.org/0000-0002-1765-9640•lucianaduques@usp.br

Contribuição: Conceituação, Análise Formal, Metodologia, Supervisão, Validação, Escrita - revisão e edição

\section{8 - Álvaro Figueredo dos Santos}

Engenheiro Florestal, Dr., Pesquisador

https://orcid.org/0000-0002-2689-9291•alvarofigueredo@gmail.com

Contribuição: Conceituação, Análise Formal, Metodologia, Supervisão, Validação, Escrita - revisão e edição

\section{Como citar este artigo}

Rezende, E. H.; Auer, C. G.; Duin, I. M.; Coelho, T. A. V.; Miranda, I. D. S.; Higa, A. R.; Silva, L. D.; Santos, Á. F. Potencial de seleção em uma população de Eucalyptus grandis Hill ex Maiden, para resistência à mancha foliar e ao cancro. Ciência Florestal, Santa Maria, v. 31, n. 4, p. 1930-1948, 2021. DOI 10.5902/1980509856935. Disponível em: https://doi.org/10.5902/1980509856935. 